\title{
Phase and power experimental study of seeded modulation instability in passive fiber ring cavities
}

\author{
Florent Bessin $^{l}$, Corentin Naveau ${ }^{1}$, Matteo Conforti ${ }^{1}$, Pascal Szriftgiser ${ }^{l}$, Alexandre Kudlinski ${ }^{l}$, and Arnaud \\ Mussot $^{l}$ \\ 1. Univ. Lille, CNRS, UMR 8523 - PhLAM - Physique des Lasers Atomes et Molécules, F-59000 Lille, France
}

Modulation instability (MI) mechanism results from the interplay between dispersion, nonlinearity and detuning in passive fiber cavities. This process allows under specific conditions ruled by a phase matching relation to a weak perturbation to grow exponentially. Most investigations have been performed from self starting MI process initiated by noise and only a few focus on coherently seeded cavities [1]. Here, we study a cavity seeded by a strong pump surrounded by two symmetric side lobes (input seeds). We numerically demonstrate that under specific relative phase values between input seed waves and input pump the parametric gain experienced by weak perturbations can vanish. Preliminary experimental results to confirm these predictions are reported.
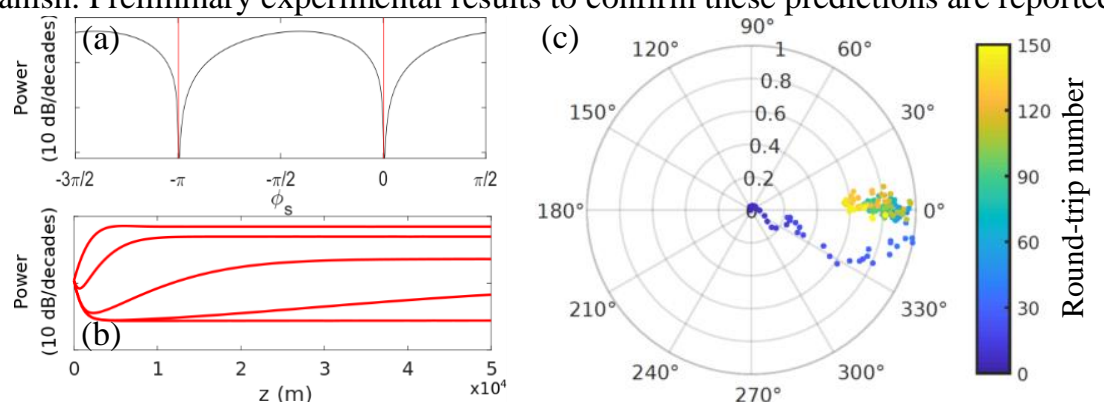

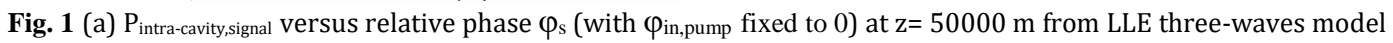
truncation (black curve). Solid vertical lines correspond to minima obtained from theory. (b) $P_{\text {intra-cavity,signal versus }}$ propagation length for various $\varphi_{\text {s. }}$ (c) Phase plane from extracted intra-cavity field (normalized signal power, relative phase signal/pump).

We studied this mechanism from an extended version of the Lugiato-Lefever equation (LLE) adding seed terms and applying a three-waves truncation [2,3]. We set seed frequencies at MI maximum gain frequencies. Many cases can be studied depending on powers and phases of input pump/seeds, but one of the most relevant is the one for which input seed fields are equal. In this case the gain is null for two specific relative phases values between input seeds/pump $\left(\varphi_{\mathrm{s}}=\varphi_{\text {in,seeds }}-\varphi_{\text {in,pump }}\right)$. We investigated these cases from numerical simulations as well as in theory for a cavity built with a $90 / 10$ coupler with a total length of $258 \mathrm{~m}$, a nonlinear coefficient of $1.2 / \mathrm{W} / \mathrm{km}$, and total losses $\alpha=0.165$. We drove the cavity in the monostable regime ( $\delta=0 \mathrm{rad})$ just above the cavity threshold $\left(\mathrm{P}_{\text {in,pump }}=0.3 \mathrm{~W}\right)$ in the anomalous dispersion region $\left(\beta_{2}\left(\omega_{\text {pump }}\right) \approx-20 \mathrm{ps}^{2} / \mathrm{km}\right)$. The ratio between the input pump

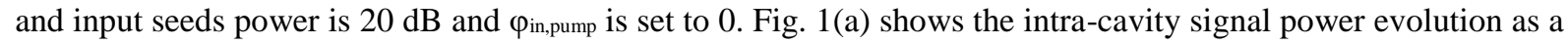
function of the relative phase $\varphi_{s}$. It is calculated from the three-waves truncated model [2,3] (black line) starting from the steady-state of the cavity. The signal power vanishes for two specific relative phase values $\left(\varphi_{\mathrm{s}} \approx-\pi\right.$ and 0 ) in very good agreement with analytical predictions calculated from a linear stability analysis of the extended LLE (red vertical lines). Fig. 1(b) shows the transient evolution of the signal power for a few specific $\varphi_{\mathrm{s}}$ values (for $\varphi_{\text {in,pump }}=0$ ). The signal can either rapidly reach its steady state value after a strong amplification stage or experience first a desamplification stage. This behaviour is quite unexpected referring to self-starting passive cavities for which the amplification is monotonous before the steady state is reached. The transient evolution of the signal phase also depends on the initial phase value (not shown here). Then, we plan to observe experimentally this phenomenon. For that purpose we built a setup inspired from Ref. [4] to measure both relative signal phase and power round trip to round trip. Preliminary results are depicted in Fig. 1(c) for a fixed $\varphi_{\mathrm{s}}$ value. The evolution of the signal in a complex plane (signal power, $\varphi_{\text {signal }}-\varphi_{\text {pump }}$ ) until a steady state is reached has been recorded. In the future, we plan to perform recordings for different $\varphi_{\mathrm{s}}$ values and to compare them with theoretical predictions

To summarize we show numerically that the gain experienced by a weak signal in a seeded passive cavity vanishes for two specific relative phase values between input seeds/pump fields and that the transient dynamics is also initial phase value dependant. Preliminary experimental results are reported.

\section{References}

[1] A. Bendahmane, J. Fatome, C. Finot, G. Millot, et B. Kibler, "Coherent and incoherent seeding of dissipative modulation instability in a nonlinear fiber ring cavity", Opt. Lett., 42, 251-254 (2017)

[2] T. Hansson, D. Modotto, and S. Wabnitz, "Dynamics of the modulational instability in microresonator frequency combs", Phys. Rev. A, 88, 023819 (2013).

[3] T. Hansson and S. Wabnitz, "Bichromatically pumped microresonator frequency combs", Phys. Rev. A, 90, 013811 (2014).

[4] A. Mussot, C. Naveau, M. Conforti, A. Kudlinski, F. Copie, P. Szriftgiser and S. Trillo, "Fibre multi-wave mixing combs reveal the broken symmetry of Fermi-Pasta-Ulam recurrence", Nat. Photonics, 12, 303 (2018). 